\title{
Impostor-based Rendering Acceleration for Virtual, Augmented, and Mixed Reality
}

\author{
Martin Mišiak \\ martin.misiak@th-koeln.de \\ University of Würzburg, HCI Group \\ Würzburg, Germany \\ TH Köln, Computer Graphics Group \\ Köln, Germany
}

\author{
Arnulph Fuhrmann \\ arnulph.fuhrmann@th-koeln.de \\ TH Köln, Computer Graphics Group \\ Köln, Germany
}

\author{
Marc Erich Latoschik \\ marc.erich.latoschik@uni- \\ wuerzburg.de \\ University of Würzburg, HCI Group \\ Würzburg, Germany
}
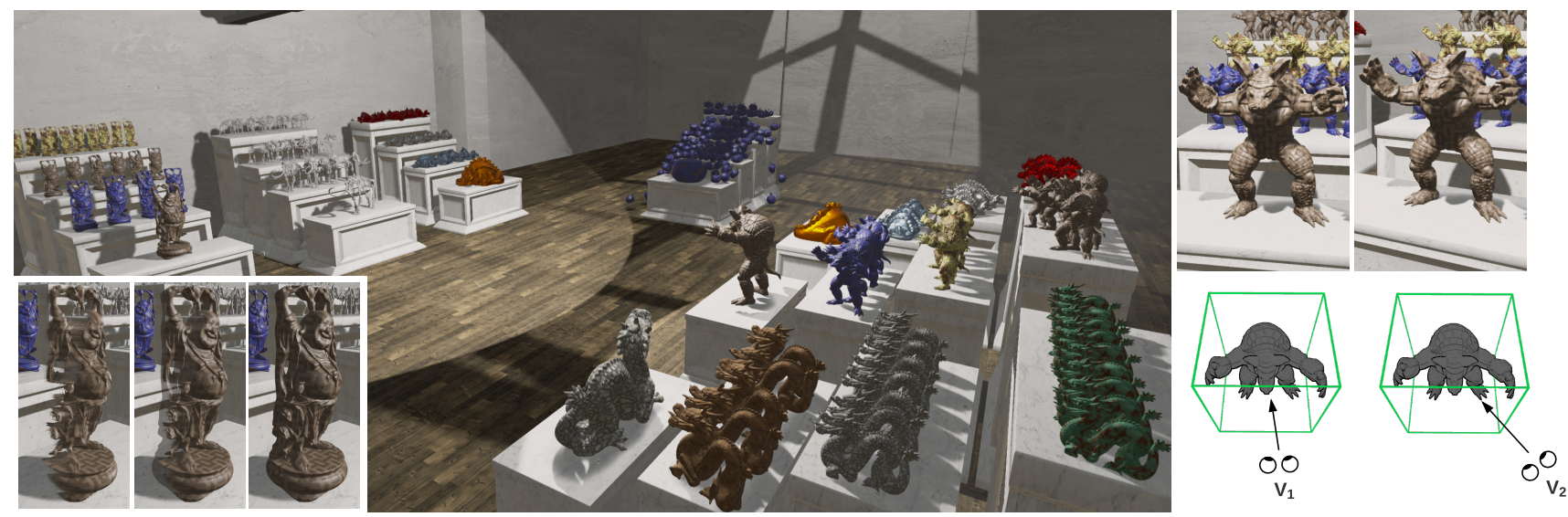

Figure 1: Middle: A scene consisting of many complex objects, each rendered using our presented impostor technique. In contrast to regular mesh rendering, our impostors allow for high performance levels for a wide variety of XR devices, while providing a similar quality level. - Right: Our impostor representation (consisting of a radiance and depth image pair for each eye) is designed for use in XR, and provides correct depth cues for a range of typical head motions. - Lower left: For larger viewpoint changes, disoccluded areas can become visible (left image). We account for this by dynamically regenerating our impostors for the novel view (right image). As new impostors become available, we perform a gradual temporal interpolation between the last two impostor images to avoid any sudden popping artifacts (middle image).

\begin{abstract}
This paper presents an image-based rendering approach to accelerate rendering time of virtual scenes containing a large number of complex high poly count objects. Our approach replaces complex objects by impostors, light-weight image-based representations leveraging geometry and shading related processing costs. In contrast to their classical implementation, our impostors are specifically designed to work in Virtual-, Augmented- and Mixed Reality scenarios (XR for short), as they support stereoscopic rendering to provide correct depth perception. Motion parallax of typical head movements is compensated by using a ray marched parallax
\end{abstract}

Permission to make digital or hard copies of part or all of this work for personal or classroom use is granted without fee provided that copies are not made or distributed for profit or commercial advantage and that copies bear this notice and the full citation on the first page. Copyrights for third-party components of this work must be honored

For all other uses, contact the owner/author(s).

VRST '21, December 8-10, 2021, Osaka, Japan

(c) 2021 Copyright held by the owner/author(s).

ACM ISBN 978-1-4503-9092-7/21/12.

https://doi.org/10.1145/3489849.3489865 correction step. Our approach provides a dynamic run-time recreation of impostors as necessary for larger changes in view position. The dynamic run-time recreation is decoupled from the actual rendering process. Hence, its associated processing cost is therefore distributed over multiple frames. This avoids any unwanted frame drops or latency spikes even for impostors of objects with complex geometry and many polygons. In addition to the significant performance benefit, our impostors compare favorably against the original mesh representation, as geometric and textural temporal aliasing artifacts are heavily suppressed.

\section{CCS CONCEPTS}

- Computing methodologies $\rightarrow$ Virtual reality; Mixed / augmented reality; Image-based rendering; Rendering.

\section{KEYWORDS}

impostors, image-based rendering, rendering acceleration

ACM Reference Format:

Martin Mišiak, Arnulph Fuhrmann, and Marc Erich Latoschik. 2021. Impostorbased Rendering Acceleration for Virtual, Augmented, and Mixed Reality. 
In 27th ACM Symposium on Virtual Reality Software and Technology (VRST '21), December 8-10, 2021, Osaka, Japan. ACM, New York, NY, USA, 10 pages. https://doi.org/10.1145/3489849.3489865

\section{INTRODUCTION}

The availability of consumer-grade affordable Augmented, Mixed, and Virtual Reality (AR, MR, and VR: XR for short) systems has generated an increased interest in the field with a large number of practical applications. From entertainment to industrial or educational applications, many use-cases profit from the specific qualities that XR can provide, e.g. high interactivity and immersion, and an increased sense of presence. A central characteristic of XR's immersive qualities is a believable depth perception, achieved by a combination of depth cues from binocular disparity, motion parallax, objects' shapes, and lighting and shading effects. The computational generation of these cues can become very expensive, especially as developments of new XR technology constantly strive to increase fields of view, screen resolutions, and refresh times. However, it is imperative, that XR applications run at high and stable framerates while exhibiting low overall latency and latency jitter [35]. Otherwise, an immersive experience can quickly deteriorate and lead to cybersickness [34, 36]. Although modern, compute based, GPU rendering pipelines are getting more efficient at rendering high polygonal geometry using triangle level culling and software rasterization [44], achieving peak performance levels still requires the reduction of geometric and shading complexities of virtual objects. In order to limit the negative impact on overall visual fidelity, objects need to retain as many of their original depth cues as possible.

A common approach for optimizing rendering performance is to pre-process the geometry with various mesh-simplification techniques [7] while preserving intricate surface shading details in image-based representations. While significant reductions are possible using these automated methods, in practice, manual adjustments are often mandatory to achieve satisfactory results. One extreme form of simplification, which relies heavily on image representations, are impostors. In its most common form, an impostor replaces the original 3D object with a rendered image of itself. This image is captured from a specific viewpoint, and mapped onto the surface of a simple quadrilateral. Due to its very simple geometry and pre-calculated radiance values, such an impostor can be rendered orders of magnitude faster than the original object. The downsides of this technique are its static nature (in terms of lighting and animation), as well as its strong view-dependent validity. Nonetheless, impostors are still a popular choice in non-XR applications, especially as a replacement for distant or contextual objects.

The use of impostors in XR on the other hand is very sparse. Analogous to other image-based rendering techniques such as normal maps, impostors fail to recreate motion parallax and binocular disparity depth cues of the underlying geometry. However, these two depth cues are central contributors to convincing depth perception in most XR systems. While a certain motion parallax error is acceptable for impostors viewed on a monoscopic screen, the absence of correct binocular disparity is particularly problematic in XR. Disparity cues generated by stereoscopic rendering immediately reveal the underlying flat quadrilateral nature of an impostor.
This is unfortunate, as XR applications are computationally very expensive and could greatly benefit from the speed-up of such a technique.

To this end, we propose a novel impostor-based acceleration technique for XR systems which are characterized by complex scenes consisting of a high number of objects. The speed-up is achieved by replacing objects with our novel stereoscopic impostor representation. Our impostors are specifically designed to work in XR, as they provide correct binocular disparity and motion parallax cues for a range of typical head movements. For larger changes in view position, our system provides a run-time impostor recreation. This process is decoupled from the main viewport rendering and can be distributed over multiple frames to ensure consistent performance. Our contributions are:

- A stereoscopic impostor representation for the use in XR. It provides correct depth perception for a range of typical head motions.

- An impostor sampling approach which outperforms mesh rendering in terms of temporal stability, as it is less prone to flickering artifacts.

- A rendering system for XR, based on the iterative regeneration of stereoscopic impostors for novel viewpoints during run-time.

\section{RELATED WORK}

\subsection{Image-based object representations}

The term impostor was first introduced in the context of complex environment walkthroughs by Maciel and Shirley [16] - "An impostor is an entity that is faster to draw than the true object, but retains the important visual characteristics of the true object”. For all intents and purposes of our paper, we define impostors as image-based object representations, which are displayed on a simple geometrical object. Impostors can be created either offline in a pre-computation step, or at run-time. Schaufler [28] presents a rendering system that relies heavily on dynamically generated impostors. Once an impostor is generated for the current view, it can be reused for multiple subsequent frames, until the viewpoint moves too far away from the original location and the impostor becomes invalid. The benefit of this approach scales with the duration an impostor can be used without requiring an update. Subsequent work has extended the dynamic impostor concept from objects to spatial hierarchies $[30,32]$.

The majority of impostor techniques rely on a pre-computed representation. By storing additional data and having larger computational budgets, the goal of most approaches is to completely replace the original geometric object. For instance, objects and even environments can be rendered as a combination of multiple simple quadrilaterals $[8,13,29]$. Storing additional per-pixel depth can be extremely beneficial for an impostor, as it can be used to generate correct motion parallax and occlusion depth cues. While earlier approaches already succeeded in this regard [21,31], it was the introduction of programmable GPU shaders that allowed for much more efficient computation of these cues using per-pixel displacement mapping [37]. With growing validity regions, another problem emerged - disocclusions. As the user moves around an impostor, areas previously not visible in the image representation 

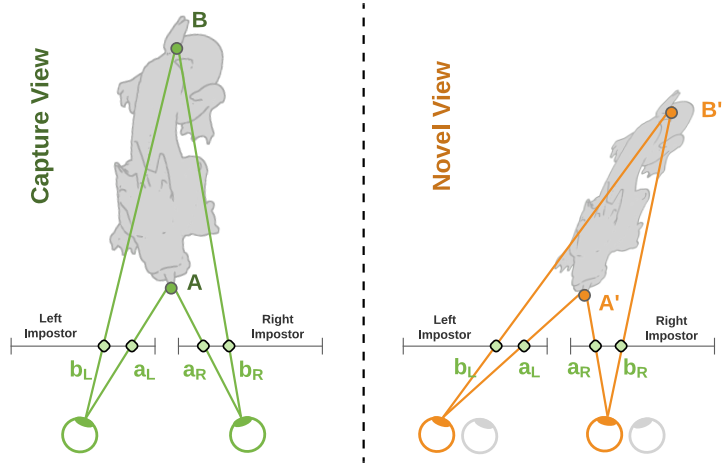

Figure 2: Stereoscopic distortions. Left: Stereoscopicimpostor as seen from the capture viewpoint. Points $A, B$ and their respective projections $a_{L, R}, b_{L, R}$ on the left and right impostor quadrilaterals. Right: When the impostor texels $a_{L, R}, b_{L, R}$ are stereoscopically fused from a novel viewpoint, the perceived location of points $A^{\prime}, B^{\prime}$ change, resulting in a distorted object.

become visible. Policarpo et al. [23, 24] extend relief mapping to multiple depth values per pixel, and use it to represent objects with wide viewing angles on a simple quadrilateral. While pre-computed impostors are typically created using an orthographic projection, other projections may be advantageous. A reverse perspective projection [4] can for instance capture oblique surface information. In this context, non-pinhole projections have also been explored to alleviate disocclusion artifacts when rendering impostors [25].

Another way of increasing impostor validity is to capture and use multiple views of an object. While this approach results in significantly higher memory requirements and longer pre-computation times, the resulting impostors are more robust against disocclusions, and capture view-dependent effects, such as specular reflections. Tecchia et al. [39] pre-compute a set of images for viewports spanning over the complete upper hemisphere. During rendering, the current view-direction is used to select the most fitting image from this set which is then displayed on a quadrilateral. A better quality can be achieved by distributing the capture positions with an icosahedron layout and interpolating between the next three closest views [40]. A variation of this technique was recently presented [6] and implemented into popular game engines [1, 2]. If viewdependent effects are not required, the number of input images can be significantly reduced by utilizing per-pixel depth information $[3,18]$.

Our impostor approach does not rely on any pre-computations. Analogous to the work of Schaufler [28], the impostors are created dynamically during run-time. Our work follows the same core principle of reusing previously rendered images of objects. However, we extend this idea to be applicable to modern XR applications.

\subsection{Image-warping systems}

Due to the decoupled impostor regeneration mechanism, our approach shares many similarities with image-warping rendering systems based on a generator/viewer architecture [33]. Such systems can be implemented locally, or in a distributed fashion where generator and viewer are on different systems. In the context of $\mathrm{XR}$, distributed architectures are used to implement low-latency streaming systems to offload the computational complexity of rendering onto a more powerful, remote device. Visibility and shading calculations can be either completely offloaded to the server $[5,12,14,15]$, or a part of visibility calculations can be performed by the client $[10,20,26]$. Local systems on the other hand provide latency reduction and framerate upsampling by reprojecting from previous frames [22, 41]. Prominent examples from the industry include SteamVR's Motion Smoothing approach and Asynchronous Space Warp from Oculus.

Most of the discussed systems can be considered orthogonal to our approach, as they operate on the entire viewport, while we are concerned with image-warping on an object level. Moreover, our approach can be used in conjunction with a viewport warping technique for additional benefit.

\section{STEREO IMPOSTORS}

To create a three-dimensional perception, an impostor has to replicate the binocular disparity between the left and right images of the substituted object. We achieve this by displaying a separate impostor for each eye. We call this impostor image-pair a stereoscopic impostor. In order to obtain a matching binocular disparity, each impostor has to be created from the viewpoint of the respective eye. The accompanying quadrilaterals, are placed in front of the object and scaled to enclose its projection. Viewing such an impostor-pair from the same position as it was created, results in a three-dimensional perception of the original object.

However, as the user moves away from the capture viewpoint, the object in the image seems to be moving with him. This apparent motion is opposite to the direction of regular parallax and can be explained by the geometrics of stereoscopic vision [11] (Figure 2). While the two images can still be stereoscopically fused together to create a depth impression, the resulting object appears distorted. The distortion is especially noticeable during movement, as the motion parallax cues of the quadrilateral are in conflict with the binocular disparity cues of the captured object. Unfortunately, viewport movement is ever so present in XR. In order to solve this issue, we consider the correct parallax movements of the depicted surface.

\subsection{Supporting Motion Parallax}

A common way of simulating surface parallax effects is by interpreting a given image as a heightfield. Surface points visible from a novel viewpoint can then be found by a number of different rendering techniques. Analogous to previous depth-based impostor approaches, we employ raymarching for determining the rayheightfield intersection point. In contrast to other methods, such as grid warping [19] or hierarchical screen-space raytracing [43], raymarching has a number of advantages for our specific use case. It operates solely on the acquired heightfield data and therefore does not require any additional data structures or pre-processing steps. This is important, as our impostors rely on a fast regeneration process. In addition, the parallax correction costs for our impostors are proportional to the distance between the novel and the capture 

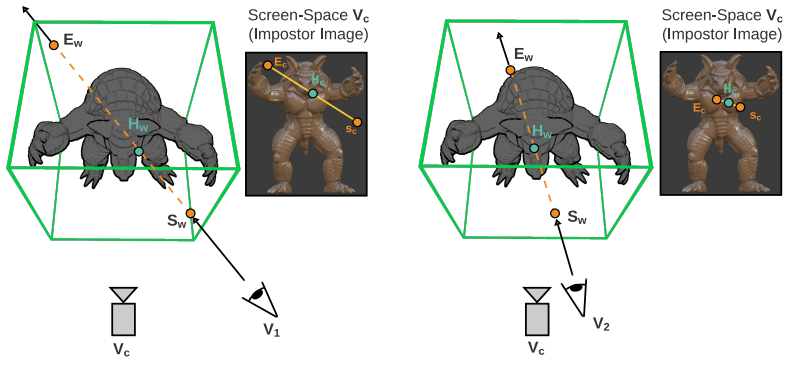

Figure 3: Motion parallax correction. To render our impostors, we first determine the entry as well as the exit point of a view ray with the object's bounding box. These coordinates are then transformed into the screen-space of the capture view $V_{c}$, where they define the search interval of the raymarch. One major characteristic of this approach is that the raymarching costs are dependent on the distance between the novel view $V_{i}$ and $V_{c}$. Views that are further away, result in a larger search interval (left), while views in the proximity of $V_{c}$ require only a few raymarch steps (right).

viewpoint. For small viewpoint changes, such as typical head sway movements or looking around, the raymarching costs are minimal. Large parallax corrections, on the other hand, are rarely necessary, as our impostors are frequently updated for novel viewpoints. The parallax correction step described in the following needs to be performed for both stereoscopic impostors separately. However, to avoid any confusion, our notation omits any left,right subscripts and describes the process exemplary for a single impostor (see Figure 3).

Given a texture coordinate $S_{c}$, the goal of the parallax correction step is to find a new coordinate $H_{c}$ along the transformed view ray $r_{c}$. This coordinate refers to the surface point on the original object $H_{w}$, which would have intersected with the world-space view ray $r_{w}$. The first step in performing a raymarch is to transform $r_{w}$ into the coordinate-space of the heightfield. For mesostructure techniques such as parallax occlusion mapping [38], this is usually the tangent-space of a triangle. For our impostors, the view ray is transformed into the screen-space of the capture camera $V_{c}$. Since we are using a perspective projection, $r_{c}$ is obtained by projecting the start $\left(S_{w}\right)$ and end point $\left(E_{w}\right)$ of $r_{w}$ into the screen-space of $V_{c}$ by multiplication with $M_{c}^{v i e w}$ and $M_{c}^{\text {proj }}$.

In order to constrain the search interval and avoid redundant raymarch steps, $S_{w}$ and $E_{w}$ should be chosen as close to the object surface as possible. We employ the object's bounding box as our impostor primitive. The starting point $S_{w}$ is then computed by simply rasterizing the bounding box, while an analytic intersection test [17] is used to determine $E_{w}$. Once $S_{c}$ and $E_{c}$ are given, the texels on this line are intersected with the view ray using a linear search, followed by a few iterations of the secant method [27]. The obtained texture coordinate $H_{c}$ of the intersection is then used to sample the impostor image.

The raymarching method employed makes the assumption of a continuous surface. This can lead to "rubber sheet" artifacts, as areas with depth discontinuities are wrongly connected. When rendering

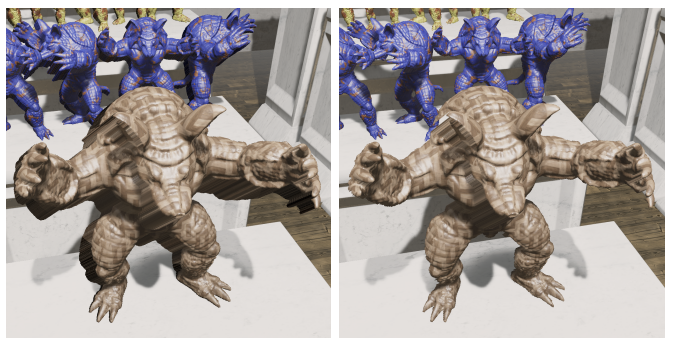

Figure 4: Left: Heightfield raymarching assumes a continuous, connected surface. This results in unwanted rubber sheets, connecting the silhouettes of an object with the background, as well as inner surfaces with large depth differences. Right: Our impostor representation allows us to safely discard silhouette rubber sheets, while keeping smaller inner surface sheets as a substitute for inpainting.

objects, this behavior can be primarily observed on silhouette edges (Figure 4, left) but also on inner object surfaces which exhibit large depth differences. Since we are raymarching on a per-object basis and use normalized depth values, background texels always have a value of 1.0. This allows us to reliably identify and discard rubber sheets in silhouette areas. Sheets spanning across inner object disocclusions are detected using a depth gradient threshold [3]. We discard surfaces with large gradients while keeping rubber sheets of smaller gradients as a performance neutral substitute for inpainting (Figure 4, right). In practice, this approach works very well for smaller view changes (e.g typical head movements). Larger view changes however lead to noticeable disocclusion errors, which can no longer be masked using inpainting. We solve this problem by dynamically regenerating our impostors for the novel view (see Section 4).

\subsection{Improving Impostor Image Quality}

In order to create a temporally stable image, the reduction of various aliasing artifacts is important. This is especially true for XR, where continuous viewport movement, as well as strong pixel magnifications amplify any existing aliasing. In the context of image-based rendering, aliasing can occur when multiple texels of the input image are projected onto one pixel of the output image. As regeneration is a core principle of our technique, the resolution of our impostors is tightly coupled to the current on-screen resolution of its underlying object. Nevertheless, aliasing can still be encountered in the time period between two regenerations, as the impostor is viewed from slightly different viewpoints. We minimize aliasing artifacts by performing run-time filtering using multiple image samples.

We evaluated different sampling strategies regarding their impact on temporal stability, as well as their performance considerations. While a naive single sample raymarch is the fastest method, the results are also very prone to temporal silhouette and inner surface aliasing. To remedy this, all impostor texels which are projected onto the footprint of a given screen pixel have to be integrated. The reference solution is therefore to perform multiple raymarches through the footprint of a given screen pixel and average the results. 

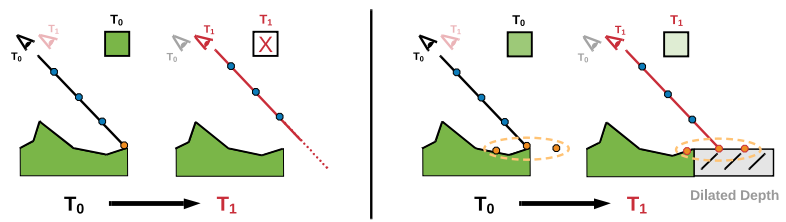

Figure 5: Silhouette edges are especially prone to temporal flickering. Left: As the head moves by small amounts between two frames, $T_{0}$ and $T_{1}$, a raymarch either hits or completely misses the surface. Right: Dilating the depth values along silhouette edges guarantees that all raymarches in the vicinity yield a hit point. Taking multiple radiance samples around this hit point improves the temporal stability of silhouettes.

However, such an approach is prohibitively expensive for a larger number of impostors.

A much cheaper alternative is to take only one raymarch through the center of the pixel, determine the hit point $H_{c}$, and average all values found in the projection of the footprint of the pixel. In contrast to the supersampling approach, we assume that each sampling ray lands in close vicinity of $H_{c}$ and does not encounter any occluding surfaces on its way. For a majority of cases, this is a very reasonable assumption, as our impostors are seldom viewed under grazing angles. When determining the size of the pixel footprint in texture-space, partial derivatives of $H_{c}$ can lead to an extreme overestimation of the actual footprint. Instead, we span a plane at the hit point location $H_{w}$, with its normal towards the capture camera $V_{c}$. Each additional sampling ray is then intersected against this plane, instead of the actual surface. The intersections are then transformed into the screen-space of $V_{c}$ and used as multi-sampling locations. While this provides adequate anti-aliasing for the object surface, it fails at silhouette edges. If the main ray closely misses the silhouette, no hit point is present and the supporting plane cannot be constructed (Figure 5, left). To remedy this issue, we dilate the depth values of our silhouettes outwards by a few pixels. This way, we can always guarantee an intersection of the view ray in the vicinity of a silhouette (Figure 5, right). Using this technique, we achieve an impostor which is temporarily stable on its surface, as well as its silhouette. The results are comparable to a supersampling solution but at a much lower computational cost.

\section{IMPOSTOR REGENERATION SYSTEM}

As we store only a single radiance and depth sample of the object's closest surface, our impostors are valid only for a small view deviation. Larger user movements will reveal disocclusions - areas not captured in the regeneration process (see Figure 1 left). Therefore, it is necessary to recreate the impostor from the novel viewpoint and hence close the disoccluded areas.

\subsection{Impostor Atlas}

To avoid performance bottlenecks using individual textures, we render and store our impostors into large atlases, each associated with one radiance and one depth texture. We use three atlases: main, history, and accumulation atlas. The accumulation atlas stores impostors during their regeneration phase. This is necessary, as impostors can regenerate over multiple frames. During this time, the current impostor is still in use and should not be replaced with a half-finished image. Once the last triangle has been rendered, the finished impostors are copied over to the main atlas which is used during regular rendering. The history atlas stores information about the previous impostor and is necessary for the blending between the current impostor image and the next. To account for different impostor sizes each atlas offers 4 different tile resolutions implemented via a quadrant subdivision approach (see appendix for details).

\subsection{Regeneration Metric}

In order to determine if an impostor is no longer valid and needs to be recreated, a regeneration metric $R$ is required. The main threats to impostor validity are disoccluded areas and resolution mismatch. Disocclusions occur when surfaces, hidden in the capture view, become visible from a novel viewpoint. While we can detect areas during the parallax correction step which are potential disocclusions, a non-conservative classification is only possible if information about the true underlying surface is present. Since our impostors store only the first visible surface layer, a precise disocclusion detection is not possible. Instead, we observe that the amount of potential disocclusion is proportional to the amount of required parallax correction. Hence, we use an estimate of parallax movement as a conservative measure for disocclusion. We employ a metric by Shade et al. [32], which measures the parallax error $P$ introduced to a flat impostor as it is viewed from a novel position. The error is computed by projecting the object's bounding box vertices $B^{i}$ onto the impostor plane. Each vertex is projected from the capture $\left(\dot{B}^{i}\right)$ as well as the novel viewpoint $\left(\ddot{B}^{i}\right)$. The angle between these two projected points is $P$. For a conservative estimate, the largest $P$ of all vertices is taken.

$$
P_{\max }=\max _{i}\left(\varangle \dot{B^{i}}, \ddot{B^{i}}\right)
$$

A regeneration due to the parallax term is only necessary when $P_{\text {max }}$ is above a certain threshold value of $\Delta P$. To account for a resolution mismatch between the object and its impostor, we introduce the texture term $T$. The resolution of the object's projected bounding box is compared to the resolution of the atlas tile, where the impostor is currently stored. If the current tile still provides the best overall fit, $T$ evaluates to 0 . If another tile from a different quadrant provides a better fit and is also free, $T$ evaluates to 1 . Note that this term does not only result in the increase of an impostor's resolution. If an impostor is stored at a higher resolution than necessary, the texture term will signal a regeneration into a lower resolution atlas tile. This in turn makes the previous high-resolution tile available for other impostors.

The parallax and texture terms form the basis of our regeneration metric. To better determine the regeneration priority when considering multiple impostors, we added two weighting terms to our metric

$$
\begin{aligned}
& D=\frac{1}{\left\|B^{c}-V\right\|} \\
& F=\frac{1}{\left\|\left(M_{v}^{\text {proj }} \cdot M_{v}^{\text {view }} \cdot B^{c}\right) \odot(1,1,0)^{T}\right\|}
\end{aligned}
$$


With $B^{c}$ being the center of the object's bounding box, and $V$ is the position of the current viewpoint. The first weighting term $D$ prioritizes objects in close proximity, while the second term $F$ weights objects based on their distance from the center of the field of view. The complete regeneration metric $R$ is then given as:

$$
R= \begin{cases}0, & \text { if } \frac{P_{\max }}{\Delta P}+T<1 \\ \left(\frac{P_{\max }}{\Delta P}+T\right) \cdot(D+F), & \text { otherwise }\end{cases}
$$

In order to suspend the regeneration process during small viewpoint movements, the metric is set to zero, if the sum of the parallax and texture term is less than 1 .

\subsection{Impostor Regeneration}

The regeneration process consists of rendering the underlying object from a novel viewpoint and storing it inside the impostor atlas. The object is rendered using any existing rendering pipeline, and therefore maintains all of its visual characteristics. In order to create impostors of more complex objects without exceeding the frame budget, the rendering process is spread over multiple frames. A subset of the object's triangles is then rasterized and shaded in each frame while the intermediary results are stored in the accumulation atlas. Once an object is completely rendered, the finished image is transferred to the main impostor atlas.

The first step of the regeneration process is to evaluate the regeneration metric for each stereoscopic impostor. The results are sorted in a priority list and the impostors most in need of regeneration are processed first. An impostor begins its regeneration process by computing its next view and projection capture matrices. The novel viewpoint for which the rendering will be performed is derived from the current head position, which is then extrapolated into the future to account for the duration of the regeneration process. As user head movements are notoriously difficult to predict, we constrain our predictions to only a few frames ahead. The predictions are made using a simple method, based on the current acceleration and velocity of the user's head-mounted display. To capture the entire object inside the impostor image, the virtual camera is rotated towards the center of the object's bounding box. The corresponding projection matrix is constructed to enclose the bounding box in its frustum. Based on a min-max projection of the bounding box, we determine the current screen resolution of the object. To maintain this resolution the impostor is assigned new texture coordinates, which point to an atlas tile with the closest matching resolution.

To keep a steady framerate, it is important that the regeneration process does not exceed a certain per-frame computational budget. We define a maximum number of per-frame regenerated triangles as our budget. While not an absolute measure of rendering time, we found it to work well enough in practice as the rendering performance is proportional to the number of triangles. In addition, the performance characteristics of a given rendering pipeline are well known in advance and can be used to estimate a "worst-case" rendering load.

As impostors are replaced by new ones, the resulting image can suffer from sudden popping artifacts, hurting the overall temporal stability. For this reason, we are performing a blending operation between the old and the new impostor image. As a new impostor is finished regenerating, the current impostor is copied over to the

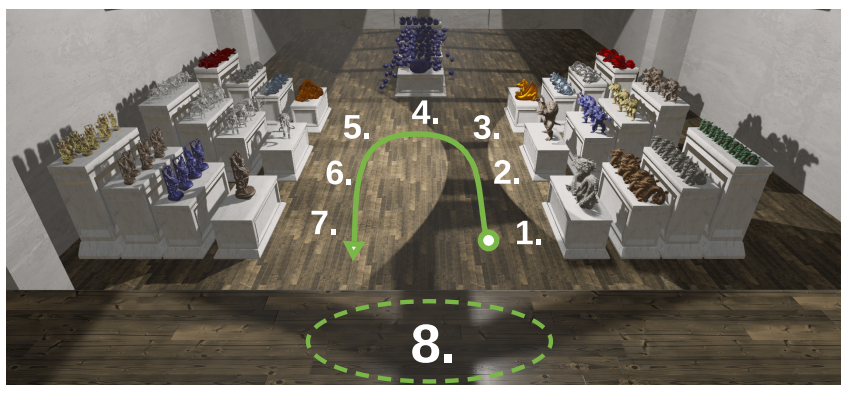

Figure 6: Top down view of our "Computer Graphics $\mathrm{Mu}$ seum" test scene. The scene contains 324 individual objects and a total amount of $83,167,576$ vertices. During the test sequence, the individual stations are visited in the order given by the numbers.

history atlas, before being replaced by the new version. After this step, the impostor is in a blending phase where both the old as well as the new impostor are rendered simultaneously. A simple linear weight determines the blending amount. Additional details, as well as an algorithmic description of our regeneration mechanism can be found in the appendix.

\section{SYSTEM EVALUATION}

Our impostor system is implemented into a custom, physically based rendering engine using OpenGL. While our technique is applicable to all XR systems, we demonstrate it in a prototypical VR application. The test system consists of an Nvidia 2070 GPU with $8 \mathrm{~GB}$ of memory, paired with a recent desktop CPU and $16 \mathrm{~GB}$ of RAM. The evaluation is performed on a stereoscopic image sequence of 8800 frames, animated using a pre-recorded camera path. We obtained the path by capturing the head position during a walk-through of our virtual test scene. To cover a broad range of rendering scenarios, we constructed a "Computer Graphics $\mathrm{Mu}$ seum" scene with 8 different stations (see Figure 6 and Table 1). Our rendering system replaces all complex objects from each station with impostors. During the sequence, the user moves from one station to the next, while inspecting the objects of each station for a short period of time ( $\sim 5$ seconds). The last station 8 is an exception to this procedure, as it shows a view of the entire test scene. The transition to this station is implemented via instantaneous teleportation of the virtual camera after the viewing of station 7 is completed.

\subsection{Visual Quality and Performance}

We evaluated the visual quality of our approach using the Structural Similarity Index Measure (SSIM) [42] and the Perceptual Similarity Metric (LPIPS) [46]. The comparisons are made against a reference sequence that uses regular mesh objects instead of impostors. All images are rendered at a resolution of $2048 \times 2048$ per eye with 4x MSAA, and asymmetric projection matrices as typically used in VR headsets. In addition, all tested methods employ view frustum culling to remove superfluous objects from the rendering process. The SSIM and LPIPS scores for each frame are evaluated separately for the left and right images. However for brevity, we present only 
Table 1: Detailed information about the individual stations in our CG Museum test scene.

\begin{tabular}{cccc}
\hline Station & Model & Number of triangles & Test case \\
\hline 1 & Stanford Dragon & $22 \times 871,414$ & Diffuse material \\
\hline 2 & Armadillo & $18 \times 345,944$ & $\begin{array}{c}\text { Dielectric material with } \\
\text { diffuse and glossy components }\end{array}$ \\
\hline 3 & EG Phlegmatic Dragon & $22 \times 677,326$ & Glossy metal material \\
\hline 4 & Utah Teapot & $199 \times 15,704$ & Large number of simple objects \\
\hline 5 & EG Phlegmatic Dragon & $22 \times 677,326$ & Highly specular metal material \\
\hline 6 & Woolly Mammoth & $20 \times 100,000$ & $\begin{array}{c}\text { Objects with very high } \\
\text { disocclusion potential }\end{array}$ \\
\hline 7 & Happy Buddha & $21 \times 1,087,716$ & $\begin{array}{c}\text { High polycount object, } \\
\text { with low disocclusion potential }\end{array}$ \\
\hline 8 & All models & $83,167,576$ & A view of the entire scene \\
\hline
\end{tabular}

the average scores. SSIM was computed using the reference Matlab implementation ${ }^{1}$ at full image resolution. LPIPS is evaluated using the reference Python implementation in version $0.1^{2}$.

The impostor system uses an atlas resolution of $8192 \times 8192$ with a $4 \times 4,10 \times 10,14 \times 14$ and $20 \times 20$ quadrant division. As the regeneration process shares the same rendering pipeline with the rest of the environment, impostors are also created using $4 \mathrm{x}$ MSAA rasterization. The parallax correction is performed using 16 steps, of which 4 steps are performed using the secant method. The radiance values around the hitpoint are multisampled using 8 samples. A budget of 250,000 triangles per-frame is used to limit impostor regeneration. The parallax term is constrained against a $\triangle P$ of 0.5 .

For performance evaluation, regular GPU time measurements are made for each frame of the sequence. All presented measurements are the sum of the left and right eye rendering times. The rendering times for our impostor technique are divided into Render Environment, Render Impostors and Generate Impostors. Although our technique does not rely on any mesh simplification steps, we also included a Level of Detail (LoD) system on top of the mesh rendering condition for a more grounded comparison. The simplified objects are reduced to $50 \%, 20 \%$, and $5 \%$ of the original primitive count and displayed based on their projected screen size.

5.1.1 Performance. When inspecting Figure 7, the performance benefit of our impostor technique can be clearly seen. For the entire duration of the sequence, the frame times of this complex scene are in the range of 7-10 ms. This ensures a consistent frame rate of $90 \mathrm{~Hz}$ on modern VR headsets. In contrast to this is the performance of regular mesh rendering. Frame times are on average around $30 \mathrm{~ms}$ and even reach $60 \mathrm{~ms}$ at station 8 , where the entire scene is in view. The use of LoDs significantly improves the mesh rendering performance. However, it is still slower by approximately a factor of 2 when compared to our impostors. This holds true even for station 8 , where all objects are rendered using the heavily reduced $5 \%$ models. Although the frame times vary greatly due to the use of frustum culling, the resulting frame rate almost never reaches the targeted $90 \mathrm{~Hz}$ for VR. Mesh rendering performs best at station 4 which consists of a large number of simple objects. The LoD version

\footnotetext{
${ }^{1}$ https://ece.uwaterloo.ca/ z70wang/research/ssim/

${ }^{2}$ https://github.com/richzhang/PerceptualSimilarity
}

even slightly outperforms the impostor technique, as the associated geometric processing costs are very minimal.

Upon closer inspection of the frame times in Figure 8, it is evident that the actual processing time of our technique in isolation is even faster. The rendering, as well as regeneration time for all impostors, takes only around 2-4 ms. The remaining majority of the frame time is taken by rendering the rest of the scene environment. Despite this fact, we still achieve an overall speed-up of $2 x-3 x$. For other $\mathrm{XR}$ systems, such as AR, the speed-up could be even higher as the rendering of the scene environment is not required.

The performance of the Render Impostors step is independent of geometric complexity and is influenced only by the number of impostors in the current frustum, as well as the number of shaded pixels. This can be seen at station 4 , where a combination of both factors is present. As a result, the measurements for the Render Impostors process are the highest during this sequence. The process itself is also view-dependent. As the user's viewpoint diverges from the last capture position, the required number of parallax-correction steps increases. This relationship however has a negligible impact on our actual performance measures, as our parallax correction is constrained to a maximum of only 16 steps.

The second half of the rendering costs associated with our technique is the regeneration of impostors which have become invalid. These costs vary depending on the geometric complexity of the underlying object, as well as the area of the currently rasterized triangle batch. Although our rendering budget, given in triangles, is sufficient to limit the regeneration costs to a specific range, a lot of variance can be observed in the individual measurements. This reflects the fact, that the rendering costs of a mesh depend on many different factors, and relying solely on the number of triangles is only a coarse approximation.

5.1.2 Image Quality. The SSIM and LPIPS scores of our impostor technique (Figure 9) are on average very high with values between $0.97-0.997$ (SSIM) and $0.005-0.017$ (LPIPS). Both scores show a very similar development over the test sequence, with LPIPS closely following SSIM. While most stations exhibit an SSIM score of 0.99 (LPIPS - 0.01), there are some notable dips in quality. Station 5 and 6 score the lowest with 0.985 (LPIPS - 0.014). This is in line with our expectations, as both stations contain objects which are challenging for our technique. Disocclusion heavy surfaces at station 6, and strong view-dependent specular highlights at station 5. Although station 3 also contains highly specular objects, it scored better than station 5 . We assume the reason lies in the sharpness of the specular highlight, as objects at station 5 exhibit a much sharper reflection.

As the virtual user moves from one station to the next, he also briefly glances at other stations in a quick look around motion. This creates dips in the scores between stations. This can be traced back to the limited regeneration budget we are using, as well as our regeneration metric. During movement, objects in near proximity and objects close to the center of the view frustum are prioritized in the regeneration process. This leaves little resources to update distant objects which are behind the user.

A similar situation occurs at the beginning of station 8, where the user is instantaneously teleported to another location. Due to this rapid change of viewpoints, disocclusions become visible. This is reflected in a lower perceptual quality score. However shortly 
Total Rendering Time

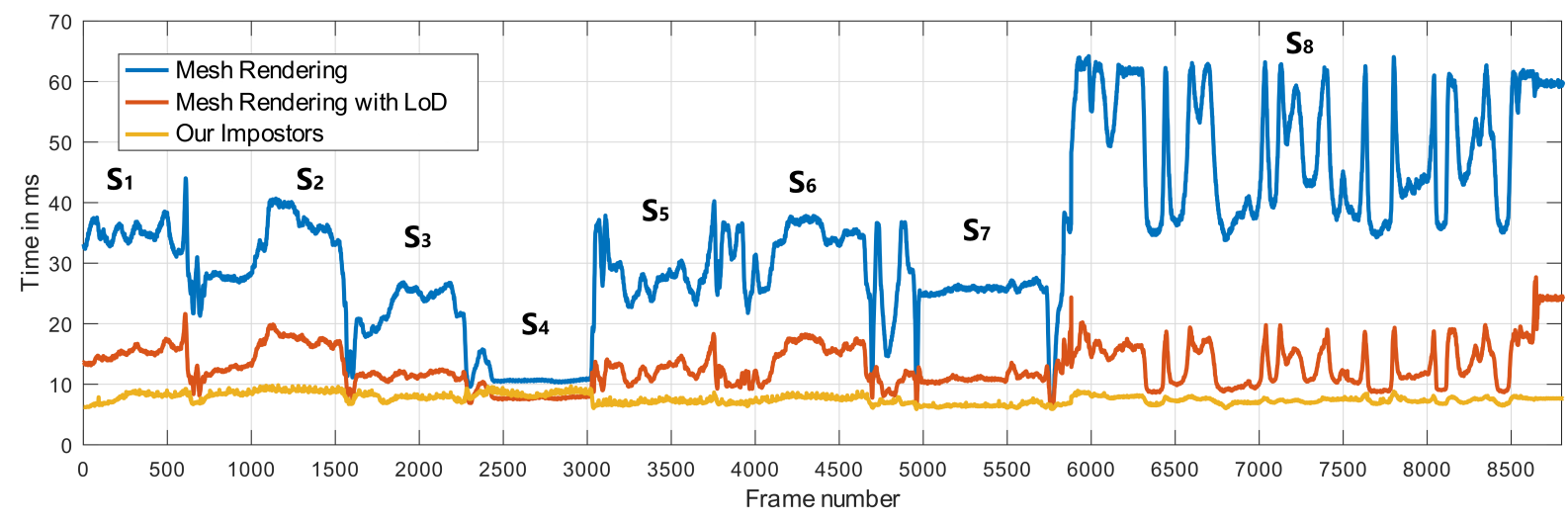

Figure 7: Total rendering time for each frame of the pre-recorded camera path measured for our impostor technique, as well as for standard mesh rendering with and without a level of detail approach. The frame numbers are associated with the visited stations by characters $S_{1}-S_{8}$.

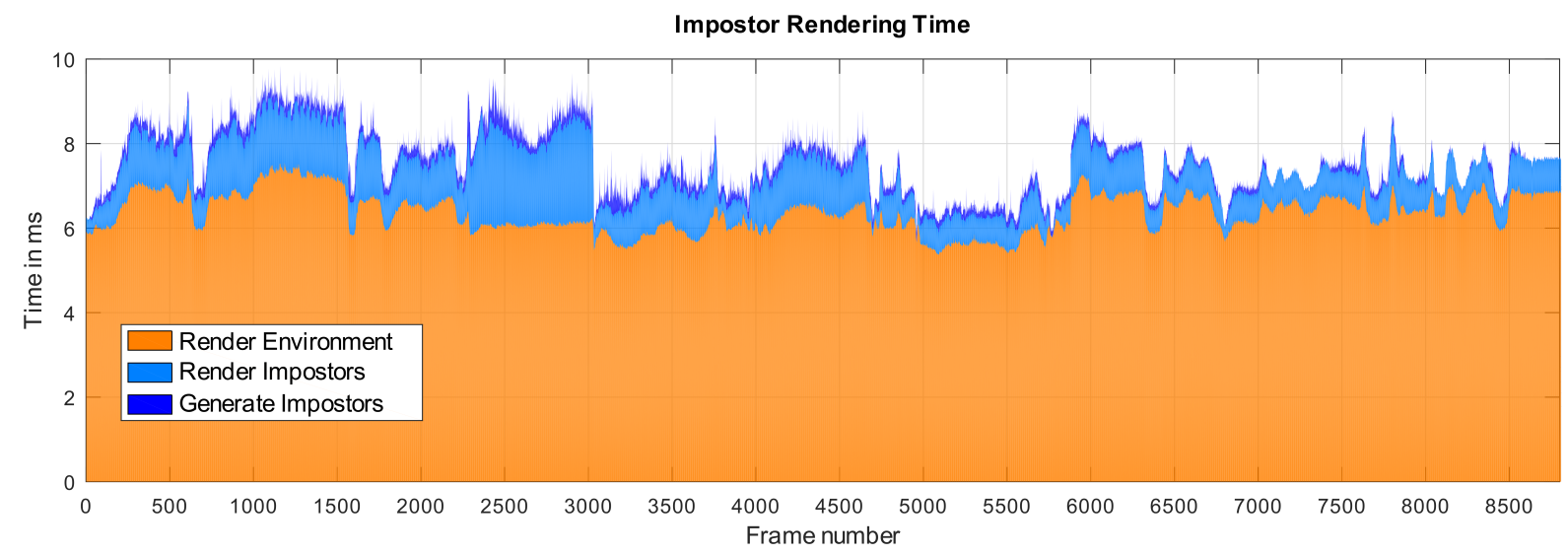

Figure 8: A detailed rendering time overview of our impostor technique during the pre-recorded camera path.

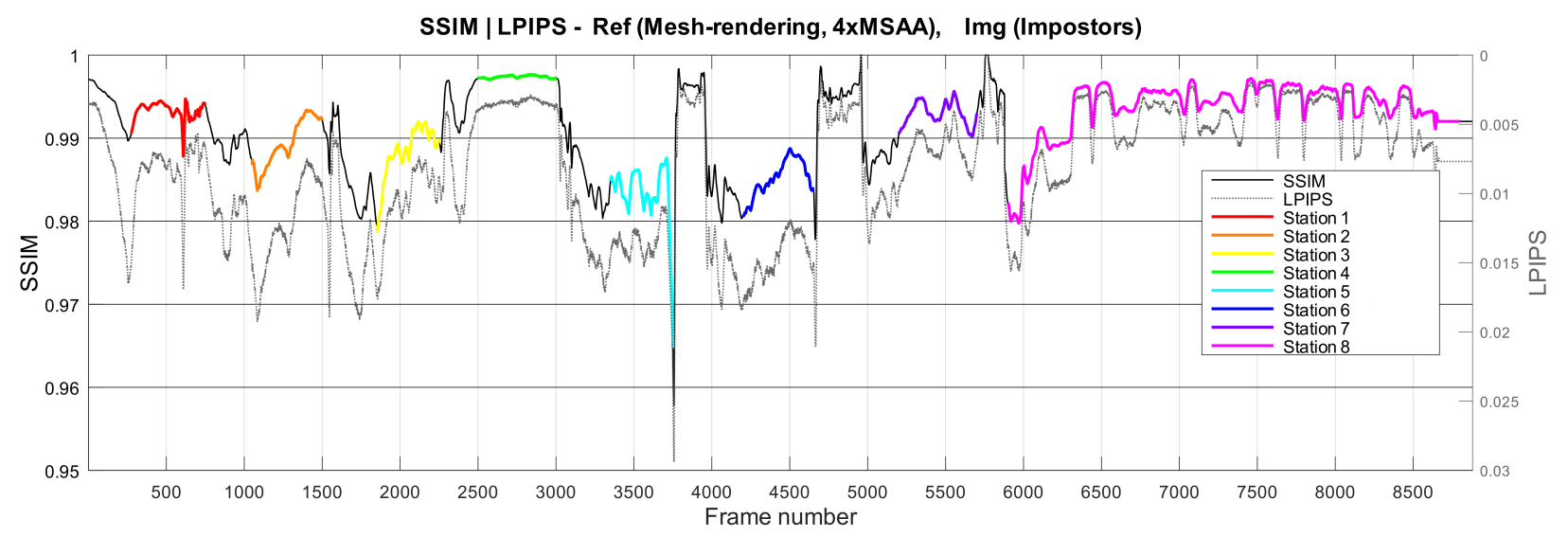

Figure 9: SSIM and LPIPS scores for our impostor technique evaluated for each frame of the pre-recorded camera path. The comparison is made against standard mesh rendering. Frames corresponding to the individual stations are color-coded only in the SSIM graph. Scores outside of the color-coded regions represent the transitions of the camera between two stations. 


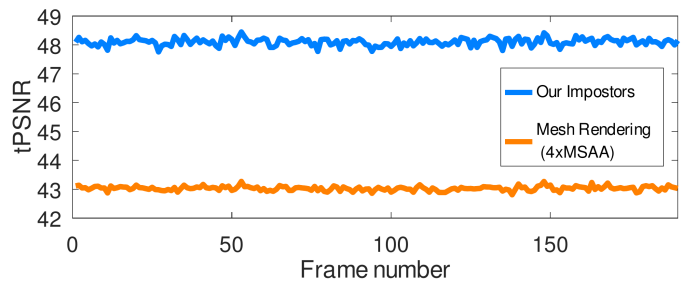

Figure 10: tPSNR scores for a camera animation sequence depicting a typical head sway motion. The camera is centered at station 6 and the objects are rendered using either our impostor technique or as regular meshes.

after, the score recovers as the impostors are regenerated for the new viewpoint. Rapid viewpoint change is an important use case, as teleportation is currently one of the main locomotion mechanisms in VR. Most practical implementations however include a small fade in/out time, which can help mask the regeneration period. For our implementation, we chose explicitly instantaneous teleportation to demonstrate a worst-case scenario.

\subsection{Temporal Stability}

While the SSIM and LPIPS image quality metrics are useful for a perceptual comparison between two images, they do not account for the temporal dependency found between the images of a sequence. This dependency however is important if we wish to analyze the impact of temporal aliasing on the perceived quality. To this end, we employ a temporal variant of the PSNR metric (tPSNR) [9], which operates on the difference images of two subsequent frames.

A sequence of 190 frames exhibiting typical head sway movements is captured and analyzed using tPSNR. The reference images are created using a 36x super-sampled mesh rendering $(12288 \times$ 12288 downsampled to $2048 \times 2048)$.

The results of our temporal stability analysis can be seen in Figure 10 (tPSNR). In comparison to regular mesh rendering using 4x MSAA and trilinear texture filtering, our impostors offer superior temporal stability with significantly fewer aliasing artifacts on object silhouettes (see appendix for further details). This is an important aspect for the overall visual quality experienced in today's head-mounted displays.

\section{LIMITATIONS}

The main limitation of our technique is its constrained support of dynamic geometry and lighting. A continuous animation or change in surface shading would require regeneration on a perframe basis, negating the performance benefit of our technique. However, we believe that our system can still be beneficial for semi-dynamic scenarios. Due to the short regeneration times, as well as no dependency on any pre-computation step, our impostors can reflect dynamic changes with a very short delay. This enables use cases with discreet lighting change, such as time of day, or interaction-based level of detail approaches, i.e., objects becoming impostors as soon as the user no longer interacts with them.

The stereoscopic nature of our impostors does guarantee the perception of highlight disparity on specular materials, as two texels corresponding to the same surface point store different radiance values. However, when the surface point is viewed from another direction, its radiance values should change accordingly. This is not the case for our impostors, where a surface point exhibits the same radiance values irrespective of the viewing direction. This creates a cue conflict as highlight disparity is present, yet the highlight itself is not view-dependent. The highlight only changes once enough viewport movement is present to trigger an impostor regeneration. According to our preliminary tests, the highlight cue conflict has only limited implications on the resulting viewing comfort of such surfaces. Nevertheless, the true severity of this issue remains unknown and should be evaluated in future user studies.

\section{CONCLUSION}

This article presents an impostor-based rendering system for XR. Our approach specifically takes important XR depth cues into account as it provides correct binocular disparity, as well as motion parallax cues for a range of typical head movements. Larger changes in view position are handled by a run-time regeneration of our impostors. This process is decoupled from the actual rendering and can be distributed over multiple frames using a user-specified frame budget. The presented rendering system allows scenes with complex objects to be rendered at high framerates on modern XR headsets and it contributes to limit latency issues potentially caused by rendering delays. The system is demonstrated on a scene with over 80 million vertices, where speed-ups of up to $6 \mathrm{x}$ have been achieved in comparison to traditional mesh rendering. In addition to the performance benefit, our impostors are also less prone to temporal aliasing artifacts, which is an important aspect for XR rendering.

We evaluated the quality of our system using the SSIM and LPIPS metrics to provide an objective and comparable measure against a ground-truth rendering approach. In future work, we additionally plan to evaluate the perceived quality based on a user study to investigate any correlation between the objective and subjective (perceived) quality. In particular, the quality of the stereoscopic depth perception, as well as the potential impact of the conflict caused by the specular highlight disparity are very interesting topics for further evaluations. We also plan to implement our impostor system into a popular game engine, as we believe it could be a useful tool for VR researchers and developers alike. To further improve our technique, we would like to explore the trade-offs between longer regeneration times and prolonged validity by storing additional information about our impostors. In addition, we wish to leverage hardware-accelerated raytracing for accurate inpainting of disoccluded areas [45], as well as for efficient selective impostor regeneration on a per-pixel basis.

\section{ACKNOWLEDGMENTS}

This work was partially funded by the German Federal Ministry of Education and Research (BMBF) under grant number 02K16C232 as part of the project Retail 4.0 as well as by the Ministry of Culture and Science of the State of North Rhine-Westphalia under grant number 005-2105-0046 as part of the project KoViTReK. 


\section{REFERENCES}

[1] 2018. Unity - Octahedral Impostors. https://github.com/xraxra/IMP. Accessed: 2021-07-05.

[2] 2020. Godot - Octahedral Impostors. https://github.com/wojtekpil/GodotOctahedral-Impostors. Accessed: 2021-07-05.

[3] Carlos Andújar, Javier Boo, Pere Brunet, Marta Fairén, Isabel Navazo, Pere Vazquez, and A Vinacua. 2007. Omni-directional Relief Impostors. Computer Graphics Forum 26, 3, 553-560.

[4] Lionel Baboud and Xavier Décoret. 2006. Rendering geometry with relief textures. In Proceedings of Graphics Interface 2006. Canadian Information Processing Society, 195-201.

[5] Kevin Boos, David Chu, and Eduardo Cuervo. 2016. Flashback: Immersive virtual reality on mobile devices via rendering memoization. In Proceedings of the 14th Annual International Conference on Mobile Systems, Applications, and Services. 291-304.

[6] Ryan Brucks. 2018. Octahedral Impostors. https://www.shaderbits.com/blog/ octahedral-impostors. Accessed: 2021-07-05.

[7] Jonathan Cohen, Marc Olano, and Dinesh Manocha. 1998. Appearance-preserving simplification. In Proceedings of the 25th annual conference on Computer graphics and interactive techniques. 115-122.

[8] Xavier Décoret, Frédo Durand, François X Sillion, and Julie Dorsey. 2003. Billboard clouds for extreme model simplification. In ACM SIGGRAPH 2003 Papers. 689696.

[9] Jon Hasselgren, Jacob Munkberg, Marco Salvi, Anjul Patney, and Aaron Lefohn 2020. Neural temporal adaptive sampling and denoising. In Computer Graphics Forum, Vol. 39. Wiley Online Library, 147-155.

[10] Jozef Hladky, Hans-Peter Seidel, and Markus Steinberger. 2019. Tessellated Shading Streaming. In Computer Graphics Forum, Vol. 38. Wiley Online Library, 171-182.

[11] Lloyd A Jeffress. 1937. Anomalous Parallax in Anaglyphs and Stereograms. The Journal of General Psychology 17, 2 (1937), 389-394.

[12] Zeqi Lai, Y Charlie Hu, Yong Cui, Linhui Sun, Ningwei Dai, and Hung-Sheng Lee. 2019. Furion: Engineering high-quality immersive virtual reality on today's mobile devices. IEEE Transactions on Mobile Computing (2019).

[13] Puneet Lall, Silviu Borac, Dave Richardson, Matt Pharr, and Manfred Ernst. 2018 View-Region Optimized Image-Based Scene Simplification. Proceedings of the ACM on Computer Graphics and Interactive Techniques 1, 2 (2018), 1-22.

[14] Kyungmin Lee, David Chu, Eduardo Cuervo, Johannes Kopf, Yury Degtyarev, Sergey Grizan, Alec Wolman, and Jason Flinn. 2015. Outatime: Using speculation to enable low-latency continuous interaction for mobile cloud gaming. In Proceedings of the 13th Annual International Conference on Mobile Systems, Applications, and Services. 151-165.

[15] Yong Li and Wei Gao. 2019. DeltaVR: Achieving high-performance mobile VR dynamics through pixel reuse. In Proceedings of the 18th International Conference on Information Processing in Sensor Networks. 13-24.

[16] Paulo WC Maciel and Peter Shirley. 1995. Visual navigation of large environments using textured clusters. In Proceedings of the 1995 symposium on Interactive 3D graphics. ACM, 95-ff.

[17] Alexander Majercik, Cyril Crassin, Peter Shirley, and Morgan McGuire. 2018. A ray-box intersection algorithm and efficient dynamic voxel rendering. Fournal of Computer Graphics Techniques Vol 7, 3 (2018).

[18] Stephan Mantler, Stefan Jeschke, and Michael Wimmer. 2007. Displacement mapped billboard clouds. In Proceedings of symposium on interactive $3 D$ graphics and games. Citeseer.

[19] William R Mark, Leonard McMillan, and Gary Bishop. 1997. Post-rendering 3D warping. In Proceedings of the 1997 symposium on Interactive 3D graphics. 7-ff.

[20] Joerg H Mueller, Philip Voglreiter, Mark Dokter, Thomas Neff, Mina Makar, Markus Steinberger, and Dieter Schmalstieg. 2018. Shading atlas streaming. ACM Transactions on Graphics (TOG) 37, 6 (2018), 1-16.

[21] Manuel M Oliveira, Gary Bishop, and David McAllister. 2000. Relief texture mapping. In Proceedings of the 27th annual conference on Computer graphics and interactive techniques. ACM Press/Addison-Wesley Publishing Co., 359-368.

[22] Edward M Peek, Burkhard C Wünsche, and Christof Lutteroth. 2014. Image warping for enhancing consumer applications of head-mounted displays. In Proceedings of the Fifteenth Australasian User Interface Conference-Volume 150 47-55.

[23] Fabio Policarpo and Manuel M Oliveira. 2006. Relief mapping of non-height-field surface details. In Proceedings of the 2006 symposium on Interactive $3 D$ graphics and games. ACM, 55-62.
[24] Fábio Policarpo, Manuel M Oliveira, and João LD Comba. 2005. Real-time relief mapping on arbitrary polygonal surfaces. In Proceedings of the 2005 symposium on Interactive $3 D$ graphics and games. ACM, 155-162.

[25] Voicu Popescu, Kyle Hayward, Paul Rosen, and Chris Wyman. 2009. Non-Pinhole Imposters. Technical Report 09-006. Purdue University.

[26] Bernhard Reinert, Johannes Kopf, Tobias Ritschel, Eduardo Cuervo, David Chu, and Hans-Peter Seidel. 2016. Proxy-guided image-based rendering for mobile devices. In Computer Graphics Forum, Vol. 35. Wiley Online Library, 353-362.

[27] Eric Risser, Musawir Shah, and Sumanta Pattanaik. 2007. Faster relief mapping using the secant method. Fournal of graphics tools 12, 3 (2007), 17-24.

[28] Gernot Schaufler. 1996. Exploiting frame-to-frame coherence in a virtual reality system. In Proceedings of the IEEE 1996 Virtual Reality Annual International Symposium. IEEE, 95-102.

[29] Gernot Schaufler. 1998. Per-object image warping with layered impostors. In Eurographics Workshop on Rendering Techniques. Springer, 145-156.

[30] Gernot Schaufler and Wolfgang Stürzlinger. 1996. A three dimensional image cache for virtual reality. In Computer Graphics Forum, Vol. 15. Wiley Online Library, 227-235.

[31] Jonathan Shade, Steven Gortler, Li-wei He, and Richard Szeliski. 1998. Layered depth images. In Proceedings of the 25th annual conference on Computer graphics and interactive techniques. 231-242.

[32] Jonathan Shade, Dani Lischinski, David H Salesin, Tony DeRose, and John Snyder. 1996. Hierarchical image caching for accelerated walkthroughs of complex environments. In Proceedings of the 23rd annual conference on Computer graphics and interactive techniques. ACM, 75-82.

[33] Ferdi Smit, Robert van Liere, Stephan Beck, and Bernd Fröhlich. 2009. An imagewarping architecture for vr: Low latency versus image quality. In 2009 IEEE Virtual Reality Conference. IEEE, 27-34.

[34] Jan-Philipp Stauffert, Kristof Korwisi, Florian Niebling, and Marc Erich Latoschik. 2021. Ka-Boom!!! Visually Exploring Latency Measurements for XR. In Extended Abstracts of the 2021 CHI Conference on Human Factors in Computing Systems (CHIEA '21). Association for Computing Machinery, New York, NY, USA.

[35] Jan-Philipp Stauffert, Florian Niebling, and Marc Erich Latoschik. 2018. Effects of Latency Jitter on Simulator Sickness in a Search Task. In 25th IEEE Conference on Virtual Reality and 3D User Interfaces (IEEE VR). 121-127.

[36] Jan-Philipp Stauffert, Florian Niebling, and Marc Erich Latoschik. 2020. Latency and Cybersickness: Impact, Causes, and Measures. A Review. Frontiers in Virtual Reality 1 (2020), 31.

[37] László Szirmay-Kalos and Tamás Umenhoffer. 2008. Displacement Mapping on the GPU-State of the Art. In Computer graphics forum, Vol. 27. Wiley Online Library, 1567-1592.

[38] Natalya Tatarchuk. 2006. Dynamic parallax occlusion mapping with approximate soft shadows. In Proceedings of the 2006 symposium on Interactive 3D graphics and games. 63-69.

[39] Franco Tecchia and Yiorgos Chrysanthou. 2000. Real-time rendering of densely populated urban environments. In Rendering Techniques 2000. Springer, 83-88.

[40] Severin Todt, Christof Rezk-Salama, Andreas Kolb, and K-D Kuhnert. 2008. GPUBased Spherical Light Field Rendering with Per-Fragment Depth Correction. In Computer Graphics Forum, Vol. 27. Wiley Online Library, 2081-2095.

[41] JMP Van Waveren. 2016. The asynchronous time warp for virtual reality on consumer hardware. In Proceedings of the 22nd ACM Conference on Virtual Reality Software and Technology. 37-46.

[42] Zhou Wang, Alan C Bovik, Hamid R Sheikh, and Eero P Simoncelli. 2004. Image quality assessment: from error visibility to structural similarity. IEEE transactions on image processing 13, 4 (2004), 600-612.

[43] Sven Widmer, D Pająk, André Schulz, Kari Pulli, Jan Kautz, Michael Goesele, and David Luebke. 2015. An adaptive acceleration structure for screen-space ray tracing. In Proceedings of the 7th Conference on High-Performance Graphics. 67-76.

[44] Graham Wihlidal. 2016. Optimizing the graphics pipeline with compute. In Game Developers Conference. 54.

[45] Niko Wißmann, Martin Mišiak, Arnulph Fuhrmann, and Marc Erich Latoschik. 2020. Accelerated Stereo Rendering with Hybrid Reprojection-Based Rasterization and Adaptive Ray-Tracing. In 2020 IEEE Conference on Virtual Reality and 3D User Interfaces (VR). IEEE, 828-835.

[46] Richard Zhang, Phillip Isola, Alexei A Efros, Eli Shechtman, and Oliver Wang. 2018. The unreasonable effectiveness of deep features as a perceptual metric. In Proceedings of the IEEE conference on computer vision and pattern recognition. 586-595. 